\title{
Enhancing community preparedness: an inventory and analysis of disaster citizen science activities
}

Ramya Chari ${ }^{1 *}$ (D), Elizabeth L. Petrun Sayers ${ }^{1}$, Sohaela Amiri², Mary Leinhos ${ }^{3}$, Virginia Kotzias ${ }^{1}$, Jaime Madrigano ${ }^{1}$, Erin V. Thomas ${ }^{3}$, Eric G. Carbone ${ }^{4}$ and Lori Uscher-Pines ${ }^{1}$

\begin{abstract}
Background: Disaster citizen science, or the use of scientific principles and methods by "non-professional" scientists or volunteers, may be a promising way to enhance public health emergency preparedness (PHEP) and build community resilience. However, little research has focused on understanding this emerging field and its implications for PHEP. To address research gaps, this paper: (1) assesses the state of disaster citizen science by developing an inventory of disaster citizen science projects; (2) identifies different models of disaster citizen science; and (3) assesses their relevance for PHEP.
\end{abstract}

Methods: We searched the English-language peer-reviewed and grey literature for disaster citizen science projects with no time period specified. Following searches, a team of three reviewers applied inclusion/exclusion criteria that defined eligible disasters and citizen science activities. Reviewers extracted the following elements from each project: project name and description; lead and partner entities; geographic setting; start and end dates; type of disaster; disaster phase; citizen science model; and technologies used.

Results: A final set of 209 projects, covering the time period 1953-2017, were included in the inventory. Projects were classified across five citizen science models: distributed or volunteer sensing $(n=19 ; 9 \%)$; contributory $(n=98$; 47\%); distributed intelligence $(n=52 ; 25 \%)$; collaborative research $(n=32 ; 15 \%)$; and collegial research $(n=8 ; 4 \%)$. Overall, projects were conducted across all disaster phases and most frequently for earthquakes, floods, and hurricanes. Although activities occurred globally, $40 \%$ of projects were set in the U.S. Academic, government, technology, and advocacy organizations were the most prevalent lead entities. Although a range of technologies were used, $77 \%$ of projects $(n=161)$ required an internet-connected device. These characteristics varied across citizen science models revealing important implications for applications of disaster citizen science, enhancement of disaster response capabilities, and sustainability of activities over time.

Conclusions: By increasing engagement in research, disaster citizen science may empower communities to take collective action, improve system response capabilities, and generate relevant data to mitigate adverse health impacts. The project inventory established a baseline for future research to capitalize on opportunities, address limitations, and help disaster citizen science achieve its potential.

Keywords: Citizen science;public health emergency preparedness, Disaster, Inventory, Disaster resilience, Emergency response, Disaster recovery

\footnotetext{
* Correspondence: rchari@rand.org

This work was supported by the US Centers for Disease Control and Prevention through research contract 200-2016-92420. The findings and conclusions in this report are those of the authors and do not necessarily represent the official position of the Centers for Disease Control and Prevention.

${ }^{1}$ RAND Corporation, 1200 South Hayes Street, Arlington, VA 22202, USA

Full list of author information is available at the end of the article
}

(c) The Author(s). 2019 Open Access This article is distributed under the terms of the Creative Commons Attribution 4.0 International License (http://creativecommons.org/licenses/by/4.0/), which permits unrestricted use, distribution, and reproduction in any medium, provided you give appropriate credit to the original author(s) and the source, provide a link to the Creative Commons license, and indicate if changes were made. The Creative Commons Public Domain Dedication waiver (http://creativecommons.org/publicdomain/zero/1.0/) applies to the data made available in this article, unless otherwise stated. 


\section{Background}

As disasters become increasingly costly due to factors such as population growth, the important role of communities and individuals in public health emergency preparedness (PHEP) has gained societal prominence [1-4]. In 2011, the Centers for Disease Control and Prevention (CDC) included community preparedness, which entailed community engagement and partnership development, as one of the core public health preparedness capabilities for state and local health departments [1]. In the same year, the Federal Emergency and Management Agency (FEMA) issued their framework on a "whole community approach" to emergency management, where multiple stakeholders (e.g., residents, community leaders, government) work together to strengthen capacities and build community resilience [2]. In the last decade, there has been growing encouragement of bystander response and the involvement of individuals in activities historically left to first responders and government (e.g. groups such as the Cajan Navy) [5, 6]. Given that disasters will always be a reality,, developing strategies for promoting community involvement in disaster preparedness should continue to be a public health and national security priority.

Against this backdrop, a citizen science movement for disaster preparedness has also emerged. Citizen science is the use of scientific principles and methods by "nonprofessional" scientists or public volunteers to explore or understand the world around them [7]. In addition to preparedness, citizen science has proliferated across scientific disciplines due to factors such as the growing accessibility of measurement and monitoring tools, ubiquity and increased computing power of mobile devices, and governmental and academic encouragement [8]. The potential benefits of disaster citizen science for improving PHEP are numerous. Through engagement in scientific activities, citizen scientists may help stretch resources and enhance governmental responses through the timely collection of local-level data. Disaster citizen science may be empowering, helping communities build social networks, develop skills, and generate data to mitigate adverse disaster impacts. Communities may therefore gain knowledge and capacity to take actions, better respond and adhere to preparedness recommendations, and increase their resilience, or ability to bounce back from disaster events.

Citizen science has a long history in fields like ecology, with discussions surrounding its uses as part of the scientific discourse [9]. In contrast, while there is an extensive literature on spontaneous and organized volunteerism in disasters, little research to date has focused on understanding the field of disaster citizen science and the use of volunteers specifically for disaster citizen science activities. As a result, there are few materials or guiding principles from which to draw lessons to support the implementation of citizen science for PHEP. Additionally, lessons learned from citizen science in other fields may not generalize to disaster settings because they are often dangerous. The chaotic environments accompanying disasters may pose risks to citizen scientists, and the integration of these activities with official response and recovery functions may not be straightforward. Therfore, research is needed to assess the overall state of disaster citizen science and draw out implications for the use and conduct of citizen science in PHEP applications. To address research gaps, we aimed to: (1) assess the state of disaster citizen science by developing an inventory of disaster citizen science projects; (2) identify and describe different models of disaster citizen science; and (3) assess implications for different disaster phases. The construction of this firstever comprehensive inventory will facilitate the identification of lessons learned that may increase the utility and value of disaster citizen science and improve system response capabilities, citizen scientist activities, and the resilience of affected communities.

\section{Methods}

To construct the disaster citizen science inventory, we: (1) developed a search strategy; (2) applied inclusion/exclusion criteria; and (3) performed data extractions and analyses.

\section{Search strategy \\ Data sources}

We reviewed the peer-reviewed and grey (e.g., white papers, technical reports) literature for disaster citizen science projects or activities (hereafter referred to as projects). As citizen science crosses a range of disciplines (e.g., ecology, sociology, biomedical, public health, engineering), we searched different databases represented multiple disciplines. For peer-reviewed literature, we searched PubMed, EBSCOhost research databases, Web of Science, Scopus, ArticleFirst, and OCLC Online Computer Library Center Electronic Collections Online. For grey literature, we searched LexisNexis, citizen science project databases and websites (see Additional file 1: Table S1), and Google (first ten pages of hits per search term). We also solicited feedback from experts and stakeholders by emailing a request for disaster-related citizen science projects on the Citizen Science Association listserv.

\section{Search terms and restrictions}

For the peer-reviewed literature, our search terms used "citizen science" terms AND "disaster" terms. For citizen science, we used multiple terms to capture the concept of non-professionals or volunteers engaging in research 
(e.g., citizen scien*, community scien*) [7]. For disasters, we included hazards identified as causing a public health emergency or a FEMA disaster declaration in the U.S. prior to 2018 [10, 11]. We also included climate change given its prominence as a national health security issue of concern [12]. (Additional file 2: Table S2) presents the complete list of search terms for the peer-reviewed literature.

Due to the large size of the grey literature, use of all citizen science terms yielded tens of thousands of returns. Therefore, for this literature, we paired each disaster term with the phrase "citizen science," "community science," or "crowdsourcing."

For all databases, we restricted searches to titles, abstracts, or keywords, and only searched English language sources. We did not restrict searches by date to be as comprehensive as possible and ensure inclusion of projects that may lend historical perspective, and our review reflects the literature published prior to 12/31/2017.

This process yielded more than 2800 articles, websites, and emails that we scanned for disaster citizen science projects. Identified projects $(n=353)$ were then screened for inventory eligibility.

\section{Inclusion/exclusion screening}

A two-step screening process was applied to the 353 projects. First, we applied a taxonomy developed by Wilderman to determine citizen science relevance for the inventory [13]. In Wilderman's taxonomy, citizen science models are characterized by volunteer engagement in the following research activities: (1) problem definition; (2) study design; (3) sample/data collection; (4) data analysis; and (5) data interpretation. We included a project if volunteers were involved in one or more of these activities, with one caveat. If volunteers "collected" data on themselves (e.g., disease symptoms) (\#3), then we also required involvement in at least one other activity for a project to be considered relevant. We chose this interpretation to guard against the inclusion of projects that involve citizens mainly as research subjects or inputs.

Second, we excluded projects that focused on routine monitoring activities (e.g., air or water quality) unless an activity was tied to a specific disaster event. Routine monitoring is vital for PHEP but raises conceptual issues about the demarcation between PHEP and routine public health functions. Therefore, we excluded monitoring projects to draw clear boundaries around disaster citizen science.

Three project team members carried out the screening process through a phased approach. Two team members applied inclusion/exclusion criteria to a set of assigned projects while the third person (the lead author) reviewed all projects and engaged in discussions to help resolve disagreements. Four rounds of screening and review occurred. By the last round of screening, reviewers had achieved a satisfactory level of agreement for including or excluding projects prior to team discussions $(87 \%$ observed proportionate agreement, kappa 0.7). Following screenings, a final set of 209 projects were eligible for the inventory.

\section{Data extraction and analysis}

We extracted the following data elements on each project (see Table 1 for more detailed descriptions): (1) project name and description; (2) lead project entities and entity type; (3) partners and other involved entities; (4) geographic setting; (5) project start and end years; (6) disaster type of focus; (7) disaster phase; (8) citizen science types; (9) citizen scientist participant roles; and (10) types of technologies used. Citizen science types and citizen scientist participant roles were considered two classification schemes for describing citizen science activities. In addition, we developed categories of project objectives through assessment across the entire dataset. We did not assign objectives to individual projects because of the difficulties of obtaining reliable information through review of project materials alone. For example, projects carried out to collect data for public health surveillance purposes may also have integrated educational or social networking components. Unless explicitly stated in existing materials however, it was not clear what project leaders would define as the intended objectives.

Four project team members performed extractions. The team used a variety of materials to obtain project information including original source documents and supplemental Google searches. Before independent extractions, the team applied a coding guide to a common set of projects $(n=15)$. The team met to discuss and resolve any differences and modify the coding guide as necessary. After trainings, three team members independently performed extractions for assigned projects while the fourth (lead author) reviewed all extractions.

\section{Results}

Fig. 1 displays the flow diagram and search results. Most of the final 209 projects were identified through Google $(n=153)$, followed by the peer-reviewed literature $(n=64)$, citizen science inventories and websites $(n=26)$, list serv responses $(n=22)$, and LexisNexis $(n=10)$. Counts include projects overlapping multiple sources. Table 2 provides a summary of the data extracted for each project. (Additional file 3: Table S3) displays the complete project inventory along with extracted data for each data element. 
Table 1 Description of extraction elements for the project inventory

\begin{tabular}{|c|c|}
\hline Element & Description and categorizations \\
\hline Project name and description & Formal name of project and description of objectives \\
\hline $\begin{array}{l}\text { Lead project entities and } \\
\text { entity type }\end{array}$ & $\begin{array}{l}\text { Lead organization(s) or individuals for the project: academic/research; government; advocacy or issues-based; community-based services; } \\
\text { volunteer or relief services; professional association; health services; technology sector; collaborative entity; individuals/loose affiliations }\end{array}$ \\
\hline $\begin{array}{l}\text { Partners and other involved } \\
\text { entities }\end{array}$ & Listing of partners or entities cited by the project (if available) \\
\hline Geographic setting & $\begin{array}{l}\text { U.S., international, or global focus. If U.S., region specified (northeast, southeast, midwest, west, southwest, national (all)). If international, } \\
\text { continent specified (North America, South America, Europe, Asia, Africa, Australia, Antarctica) }\end{array}$ \\
\hline Project start and end years & Official year of project launch and end year (or ongoing) \\
\hline Disaster type & $\begin{array}{l}\text { Disaster(s): accidental explosion/fire; harmful algal bloom/cyanobacteria; drought; earthquake; flood; chemical contamination; hurricane/ } \\
\text { typhoon/cyclone; disease outbreak; technological failure; mud/landslide; nuclear radiation; severe storm/weather; terrorism; tornado; } \\
\text { tsunami; volcanic activity; wildfire; all hazards; other }\end{array}$ \\
\hline Disaster phase & $\begin{array}{l}\text { Preparedness (pre-disaster, prevention and preparation activities dominate); response (during or in the immediate aftermath of the } \\
\text { disaster, crisis activities dominate); recovery (post-disaster, rebuilding activities dominate); all phases }\end{array}$ \\
\hline Citizen science types & $\begin{array}{l}\text { Citizen science type based on the level of volunteer involvement: } \\
\text { o Contributory. Volunteers involved mainly in data collection or reporting for projects led by professional scientists. } \\
\text { o Collaborative or co-created. Volunteer and professional scientists working together on many aspects of the research for projects led by } \\
\text { either group. } \\
\text { o Collegial. Volunteers leading all aspects of the research with little participation by professional scientists. }\end{array}$ \\
\hline $\begin{array}{l}\text { Citizen scientist participant } \\
\text { roles }\end{array}$ & Roles: (1) data collectors or reporters; (2) data interpreters and/or analyzers; and/or (3) problem definition and/or study design \\
\hline Type of technologies used & $\begin{array}{l}\text { Technologies used by volunteers: internet-connected device; communication device (e.g., phone, text, fax, radio); online forms/survey tools; } \\
\text { crowdsourcing reporting applications (allows users to report or submit information); crowdsourcing analytical applications (allows users to } \\
\text { engage in analytical tasks); mapping platforms/technologies; camera/video; sampling equipment/monitors/sensors; do-it-yourself sampling } \\
\text { equipment; analytical software or tools; none; other; unknown }\end{array}$ \\
\hline
\end{tabular}

${ }^{a}$ Framework for citizen science type adopted from: Shirk et al. [14]

\section{Disaster citizen science project objectives}

Overall, the disaster citizen science projects reviewed in this study were designed to achieve many different objectives, including: assessment of risks or community vulnerabilities; surveillance, early-warning, and monitoring; database or repository building; historical research or baseline establishment; intervention development and testing; epidemiological investigations; and population needs assessments. In addition, beyond scientific objectives, projects could also be designed to achieve broader societal impacts that may yield benefits for enhancing community resilience such as performing outreach to isolated groups, providing education and raising awareness about hazards and impacts, or building networks through collaborative problem-solving [15].

\section{Disaster citizen science models}

The two classification schemes describing citizen science activities together comprised a framework incorporating elements of typologies developed by Shirk et al. [14] and Haklay [16]. Using the new framework,

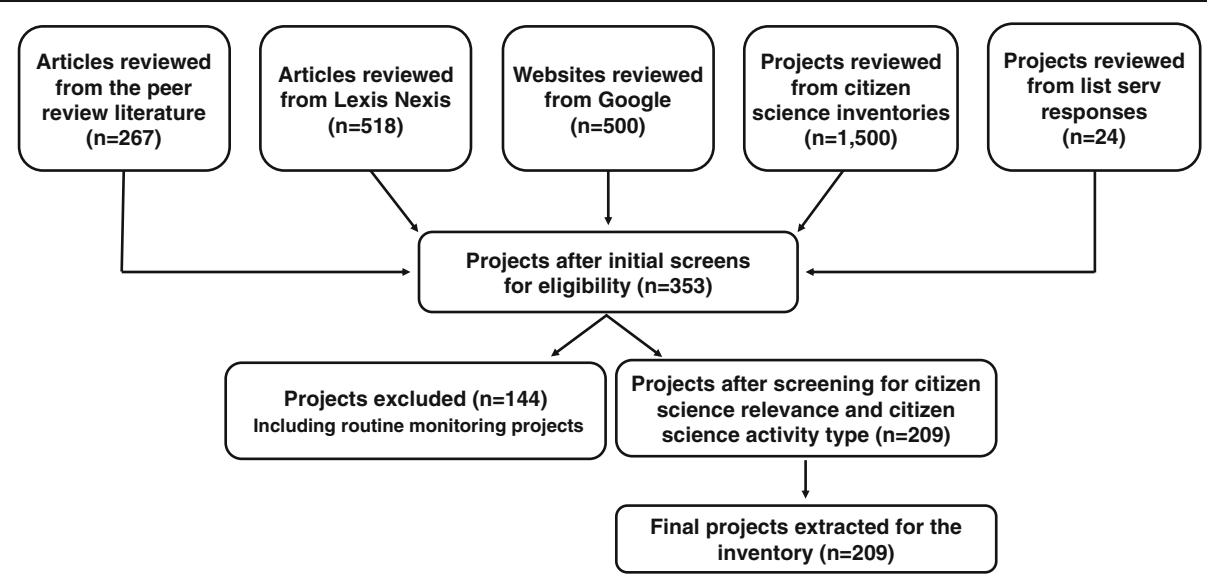

Fig. 1 Flow diagram for project inventory development. Following eligibility review of over 2800 articles, websites, and potential projects, 353 potential projects were identified. Screening for citizen science relevance and removal of monitoring projects resulted in 209 projects included in the final inventory 
Table 2 Disaster citizen science projects grouped by citizen science model

\begin{tabular}{|c|c|c|c|c|}
\hline Project name & Description of citizen science or volunteer activities & Location & Dates & Disaster \\
\hline \multicolumn{5}{|l|}{ Distributed sensing projects $(n=19)$} \\
\hline \multicolumn{5}{|l|}{ Seismometers in school projects } \\
\hline 1. IRIS networks & Create an international seismometer network in K-16 classrooms & Global & 2001- & EQ \\
\hline 2. Nera Project & Create a European network of school seismometers & Global & 2011- & EQ \\
\hline $\begin{array}{l}\text { 3. Jamaican Educational Seismic } \\
\text { Network }\end{array}$ & $\begin{array}{l}\text { Understand Jamaica's seismic risk through school-based seismic } \\
\text { network }\end{array}$ & N Am & 2016- & EQ \\
\hline 4. EduSeis & $\begin{array}{l}\text { Create a school-based earthquake monitoring system in four European } \\
\text { countries }\end{array}$ & Europe & 1996- & EQ \\
\hline 5. O3EProject & Create a seismometer network in European schools & Europe & $\begin{array}{l}2007- \\
09\end{array}$ & EQ \\
\hline 6. Seismo at School & Create a seismometer network in Swiss schools & Europe & 2007- & EQ \\
\hline $\begin{array}{l}\text { 7. Seismology in Schools } \\
\text { (Seismeolaíocht sa Scoil) }\end{array}$ & Create a seismic network of primary and secondary educational sectors & Europe & 2007- & EQ \\
\hline 8. SISMOS à l'Ecole & $\begin{array}{l}\text { Place seismometers in schools to record regional or global seismic } \\
\text { activity }\end{array}$ & Europe & 2006- & EQ \\
\hline 9. UK School Seismology Project & School network detects and shares seismometer measurements & Europe & 2007- & EQ \\
\hline 10. Seismometers in Schools & Create a seismometer network in Australian schools & Australia & 2012- & EQ \\
\hline 11. Seismometers in Schools & Create a seismometer network in New Zealand schools & Australia & 2013- & EQ \\
\hline 12. MiQuakes & Place seismometers in Michigan schools & US & 2011- & EQ \\
\hline 13. Oregon Shakes & Place seismometers in Oregon schools & US & Current & EQ \\
\hline 14. Princeton Earth Physics Project & Pioneer the creation of seismic networks in U.S. schools & US & 1994 & EQ \\
\hline \multicolumn{5}{|l|}{ Other projects } \\
\hline 15. MyShake & $\begin{array}{l}\text { Create seismic network using smartphone sensors to report earthquake } \\
\text { shaking }\end{array}$ & Global & 2016- & EQ \\
\hline 16. Quake-Catcher Network & Sense seismic motion through a computer-connected sensor network & Global & 2008- & EQ \\
\hline 17. Community Seismic Network & $\begin{array}{l}\text { Monitor earthquakes through a computer-connected network of } \\
\text { sensors }\end{array}$ & US & 2011- & EQ \\
\hline 18. NetQuakes & Install seismographs in areas with broadband internet connection & US & 2009- & EQ \\
\hline 19. weather@home & $\begin{array}{l}\text { Run regional climate modeling experiments on network of volunteer } \\
\text { computers }\end{array}$ & Global & 2010- & $\mathrm{CL}$ \\
\hline \multicolumn{5}{|l|}{ Contributory projects $(n=98)$} \\
\hline \multicolumn{5}{|l|}{ Seismic surveys } \\
\hline 1. LastQuake (International) & Report earthquake observations through online or mobile applications & Global & 2014- & EQ \\
\hline $\begin{array}{l}\text { 2. Have You Felt an Earthquake, } \\
\text { UK }\end{array}$ & & Europe & 2003 & EQ \\
\hline $\begin{array}{l}\text { 3. Other European countries }(n= \\
\text { 26) }\end{array}$ & & Europe & Current & EQ \\
\hline 4. Felt It (New Zealand) & & Australia & 2001- & EQ \\
\hline 5. Did You Feel It? (US) & & US & 1997- & EQ \\
\hline \multicolumn{5}{|l|}{ Other projects } \\
\hline $\begin{array}{l}\text { 6. Impacts of } 2010 \text { Haiti } \\
\text { earthquake }\end{array}$ & Community workers conduct health surveys in the Haitian diaspora & US & 2010 & EQ \\
\hline $\begin{array}{l}\text { 7. Cazadores de Crecidas, } \\
\text { Argentina }\end{array}$ & $\begin{array}{l}\text { Estimate river discharges through videos and photos to help flood } \\
\text { modeling }\end{array}$ & S Am & 2014- & $\mathrm{FL}$ \\
\hline 8. CITHYD (Citizen Hydrology) & Collect water level data in Italian waterbodies & Europe & 2016- & $\mathrm{FL}$ \\
\hline 9. FloodCrowd & Report floods and impacts & Europe & 2015- & $\mathrm{FL}$ \\
\hline 10. The FloodScale Project & $\begin{array}{l}\text { Provide or share home movies of flooding to use in modeling flash } \\
\text { floods }\end{array}$ & Europe & $\begin{array}{l}2012- \\
15\end{array}$ & $\mathrm{FL}$ \\
\hline 11. The RiskScape Project & Provide photo reports of floods to develop flood hazard models & Australia & 2014 & $\mathrm{FL}$ \\
\hline 12. Community flood monitoring & Install rain gauges in volunteer homes & Asia & 2009 & $\mathrm{FL}$ \\
\hline 13. Flood hazard mapping, India & Use participatory mapping approaches to assess flood vulnerability & Asia & -2014 & $\mathrm{FL}$ \\
\hline 14. Flood Patrol (UP-NOAH) & Report floods and impacts to inform preparedness efforts, Philippines & Asia & 2012- & $\mathrm{FL}$ \\
\hline 15. Jakarta floods (PetaJakarta) & Report flood events using social media & Asia & 2014- & $\mathrm{FL}$ \\
\hline
\end{tabular}


Table 2 Disaster citizen science projects grouped by citizen science model (Continued)

\begin{tabular}{|c|c|c|c|c|}
\hline Project name & Description of citizen science or volunteer activities & Location & Dates & Disaster \\
\hline & & & 15 & \\
\hline $\begin{array}{l}\text { 16. Flood, water monitoring, } \\
\text { Kenya }\end{array}$ & Measure water levels in Sondu River basin, Kenya & Africa & $2014-$ & $\mathrm{FL}$ \\
\hline 17. FLOCAST & Enable citizen flood reports to improve flash-flood predictions & US & 2013- & $\mathrm{FL}$ \\
\hline 18. Citizen science for the El Nino & Report coastal flood impacts due to the 2015-2016 El Nino & US & $\begin{array}{l}2015- \\
16\end{array}$ & $\mathrm{FL}$ \\
\hline 19. Boulder flood & Use of crowdsourcing map to report flood/damage observations & US & 2013 & $\mathrm{FL}$ \\
\hline 20. Crowdmap & $\begin{array}{l}\text { Post observations about geological exposures or hazards to online } \\
\text { community }\end{array}$ & Europe & $2011-$ & FL,LS \\
\hline 21. Crowdwater & $\begin{array}{l}\text { Provide flood, drought reports to improve forecasts of hydrological } \\
\text { events }\end{array}$ & Europe & $2016-$ & $\mathrm{FL}, \mathrm{DR}$ \\
\hline $\begin{array}{l}\text { 22. Drought Information } \\
\text { Supported by Citizen Scientists } \\
\text { (DISCS) }\end{array}$ & $\begin{array}{l}\text { Provide hydrologic and agricultural information to the scientific } \\
\text { community }\end{array}$ & US & $2017-$ & DR \\
\hline 23. DroughtWatch & Support local drought vulnerability assessments through reporting data & US & $\begin{array}{l}2009- \\
14\end{array}$ & DR \\
\hline 24. King Tides Project International & Report King Tides observations to understand flood risk in coastal areas & Global & 2009- & $\mathrm{FL}, \mathrm{CL}$ \\
\hline 25. MyCoast King Tides $(n=9)$ & $\begin{array}{l}\text { Document King Tides to track sea level rise and impacts (in nine US } \\
\text { states) }\end{array}$ & US & 2014- & $\mathrm{FL}, \mathrm{CL}$ \\
\hline 26. Urban Tides Initiative & Report tide observations to understand effects of sea level rise & US & 2015- & $\mathrm{FL}, \mathrm{CL}, \mathrm{SW}$ \\
\hline 27. Phones and Drones & $\begin{array}{l}\text { Provide photos, videos of coastal damage due to the 2015-16 El Nino } \\
\text { in CA }\end{array}$ & US & 2016 & $\mathrm{FL}, \mathrm{CL}, \mathrm{SW}, \mathrm{HR}$ \\
\hline 28. MyCoast StormReporter $(n=6)$ & Report coastal storm damages (in six US states) & US & 2014- & $\mathrm{FL}, \mathrm{SW}, \mathrm{HR}$ \\
\hline 29. mPING & $\begin{array}{l}\text { Collect weather information to improve weather predictions and } \\
\text { forecasting }\end{array}$ & Global & $2012-$ & $\begin{array}{l}\mathrm{FL}, \mathrm{SW}, \mathrm{HR} \\
\mathrm{TD}, \mathrm{LS}\end{array}$ \\
\hline 30. SKYWARN & $\begin{array}{l}\text { Trained weather spotters report data to improve emergency warning } \\
\text { services }\end{array}$ & US & 1960- & $\mathrm{FL}, \mathrm{SW}, \mathrm{HR}, \mathrm{TD}$ \\
\hline $\begin{array}{l}\text { 31. Community Collaborative Rain, } \\
\text { Hail, and Snow Network }\end{array}$ & Measure precipitation for drought and flood modeling and monitoring & US & 1998- & $\mathrm{FL}, \mathrm{SW}, \mathrm{DR}$ \\
\hline 32. Community DustWatch & Monitor wind erosion and dust events in Australia & Australia & $2002-$ & SW \\
\hline 33. Send Us your Dirt from Sandy & $\begin{array}{l}\text { Send researchers dirt samples post-Superstorm Sandy for chemical } \\
\text { analysis }\end{array}$ & US & $\begin{array}{l}2012- \\
15\end{array}$ & $H R$ \\
\hline 34. SkyTruth Spill Tracker & $\begin{array}{l}\text { Report pollution incidents from hurricanes occurring during the fall of } \\
2017\end{array}$ & US & $2017-$ & HR \\
\hline 35. Waterisotopes.org & Collect precipitation during Superstorm Sandy and send to researchers & US & $\begin{array}{l}2012- \\
13\end{array}$ & $\mathrm{HR}$ \\
\hline 36. El Reno tornado survey & $\begin{array}{l}\text { Contribute observations (photos, videos, visual reports) of the El Reno } \\
\text { tornado }\end{array}$ & US & $\begin{array}{l}2013- \\
15\end{array}$ & TD \\
\hline 37. Report a Landslide & Provide reports of landslide observations in Great Britain & Europe & 2008- & LS \\
\hline 38. Did You See It? & Contribute to a US database of landslide observations & US & $\begin{array}{l}2012- \\
16\end{array}$ & LS \\
\hline 39. Bloomin' Algae! & Report HABs in the United Kingdom & Europe & $2017-$ & $\mathrm{HAB} / \mathrm{CB}$ \\
\hline 40. Algae Alert Network & Monitor for HABs in the St. Croix River, WI & US & $2012-$ & $\mathrm{HAB} / \mathrm{CB}$ \\
\hline 41. bloomWatch & Report cyanobacteria blooms & US & 2010- & $\mathrm{HAB} / \mathrm{CB}$ \\
\hline 42. Tracking algal blooms & Engage pilots to photo algal blooms in Lake Erie & US & 2016- & $\mathrm{HAB} / \mathrm{CB}$ \\
\hline 43. CyanoTRACKER & $\begin{array}{l}\text { Facilitate public reports of cyanobacteria blooms in Georgia } \\
\text { waterbodies }\end{array}$ & US & 2015- & $\mathrm{HAB} / \mathrm{CB}$ \\
\hline 44. HAB Watch & Create a HAB monitoring network in Southern California & US & 2011- & $\mathrm{HAB} / \mathrm{CB}$ \\
\hline 45. Measure the Muck & $\begin{array}{l}\text { Measure waterbody contaminants after flooding that contribute to } \\
\text { HABs }\end{array}$ & US & 2017 & $\mathrm{HAB} / \mathrm{CB}$ \\
\hline 46. Smith River algae reports & Visitors photograph and report algae growth in the Smith River, MT & US & $2017-$ & $\mathrm{HAB} / \mathrm{CB}$ \\
\hline 47. Owasco Lake HAB monitoring & Monitor and sample for HABs in Owasco Lake, NY & US & 2015- & $\mathrm{HAB} / \mathrm{CB}$ \\
\hline 48. HAB monitoring (Multiple) & $\begin{array}{l}\text { Integrate HAB monitoring into regular water quality monitoring } \\
\text { activities }\end{array}$ & US & Current & $\mathrm{HAB} / \mathrm{CB}$ \\
\hline 49. Forest fuels measurement & Report data on forest fuels observations for wildfire risk prediction & N Am & $2012-$ & WF \\
\hline
\end{tabular}


Table 2 Disaster citizen science projects grouped by citizen science model (Continued)

\begin{tabular}{|c|c|c|c|c|}
\hline Project name & Description of citizen science or volunteer activities & Location & Dates & Disaster \\
\hline 50. Live fuel moisture monitoring & Measure moisture content in living plant tissue to predict wildfire risk & US & $2013-$ & WF \\
\hline 51. Is Ash Falling? & Collect ashfall samples during volcanic eruptions & Global & 2013- & $\mathrm{VL}$ \\
\hline 52. myVolcano & Collect ash samples during volcanic eruptions and report observations & Europe & 2010- & $\mathrm{VL}$ \\
\hline 53. Global Mosquito Alert & Enact global surveillance and control of mosquito species & Global & $2017-$ & $\mathrm{DO}$ \\
\hline 54. MosquitoWEB & Observe and send mosquitoes to researchers in Portugal & Europe & 2014- & DO \\
\hline 55. Muggenradar (Mosquito Radar) & Observe and send mosquitoes to researchers in the Netherlands & Europe & 2014- & DO \\
\hline 56. Animal mortality monitoring & Monitor and report animal deaths to prevent Ebola outbreaks & Africa & $\begin{array}{l}2001- \\
03\end{array}$ & DO \\
\hline 57. Oil Reporter & Report observations of oil spill hazards & US & $\begin{array}{l}2010- \\
11\end{array}$ & $\mathrm{CH}$ \\
\hline 58. Oil Spill Tracker & Report and track impacts of the Deepwater Horizon oil spill & US & $\begin{array}{l}2010- \\
17\end{array}$ & $\mathrm{CH}$ \\
\hline $\begin{array}{l}\text { 59. Integrated Fukushima Ocean } \\
\text { Radionuclide Monitoring Network }\end{array}$ & Monitor Canada's oceans for radionuclides through seawater sampling & N Am & 2014- & NR \\
\hline 60. Our Radioactive Ocean & Collect seawater samples to monitor radiation levels & US & $2013-$ & NR \\
\hline \multicolumn{5}{|l|}{ Distributed intelligence $(n=52)$} \\
\hline $\begin{array}{l}\text { 1. Digital humanitarian projects }(n= \\
\text { 34) }\end{array}$ & $\begin{array}{l}\text { Support disaster response efforts in real-time through analyzing large } \\
\text { amounts of different types of data. Includes } 34 \text { deployments. }\end{array}$ & $\begin{array}{l}\text { N Am, S Am, Europe, Asia, } \\
\text { Australia, US, Africa, Oceania }\end{array}$ & $\begin{array}{l}2010- \\
17\end{array}$ & $\begin{array}{l}\mathrm{EQ}, \mathrm{FL}, \mathrm{HR}, \\
\mathrm{DO}, \mathrm{VL}, \mathrm{TR}, \\
\mathrm{DR}\end{array}$ \\
\hline $\begin{array}{l}\text { 2. Fukushima Futaba } 2011 \text { Archive of } \\
\text { Japan Disasters }\end{array}$ & $\begin{array}{l}\text { Preserve memories of affected communities in Futaba, Japan and foster } \\
\text { research }\end{array}$ & Asia & 2013- & $E Q, N R, T S$ \\
\hline $\begin{array}{l}\text { 3. SHETRAN and River Watch group } \\
\text { catchment monitoring }\end{array}$ & $\begin{array}{l}\text { Implement community flood observation program in northeast England } \\
\text { to support development of a catchment model }\end{array}$ & Europe & $\begin{array}{l}2013- \\
16\end{array}$ & $\mathrm{FL}$ \\
\hline 4. Storm Photo & Document and determine severity of flooding in California & US & 2015- & $\mathrm{FL}$ \\
\hline 5. WeSenselt & Create citizen flood observatories through use of sensor devices & Europe & $\begin{array}{l}2012- \\
16\end{array}$ & $\mathrm{FL}, \mathrm{CL}, \mathrm{DR}$ \\
\hline 6. Operation Weather Rescue & Transcribe old weather observations for climate modeling & Europe & $2017-$ & SW \\
\hline 7. iCoast - Did the Coast Change? & Identify coastal changes following extreme storms & US & 2014- & $\mathrm{SW}, \mathrm{HR}$ \\
\hline 8. CycloneCenter & Estimate intensity of cyclones through satellite images & Global & 2012- & $\mathrm{HR}$ \\
\hline $\begin{array}{l}\text { 9. Agricultural recovery post- } \\
\text { Hurricane Mitch }\end{array}$ & $\begin{array}{l}\text { Enlist Nicaraguan farmers in assessing farming methods that could } \\
\text { enhance disaster recovery }\end{array}$ & S Am & 1999 & HR \\
\hline 10. Rural Alaska Monitoring Program & Community monitoring for climate-mediated health threats & US & 2014- & $\mathrm{CL}, \mathrm{HAB} / \mathrm{CB}$ \\
\hline 11. cyanoMonitoring & Monitor cyanobacteria populations over time & US & 2010- & $\mathrm{HAB} / \mathrm{CB}$ \\
\hline 12. cyanoScope & Understand where and when cyanobacteria species occur & US & $2010-$ & $\mathrm{HAB} / \mathrm{CB}$ \\
\hline 13. SoundToxins & Explore Puget Sound conditions that affect algal bloom events & US & 2006- & $\mathrm{HAB} / \mathrm{CB}$ \\
\hline $\begin{array}{l}\text { 14. National Phytoplankton } \\
\text { Monitoring Network }\end{array}$ & Monitor marine phytoplankton and algal blooms across the US & US & 2001- & $\mathrm{HAB} / \mathrm{CB}$ \\
\hline 15. Community volcano monitoring & Create network for volcano monitoring in Ecuador & S Am & 2000 & $\mathrm{VL}$ \\
\hline 16. Mosquito Habitat Mapper & Track mosquito larvae, eliminate breeding sites, and share data & Global & $2017-$ & $\mathrm{DO}$ \\
\hline 17. Mosquito Alert & Track mosquitos, breeding sites, and validate shared photos & Europe & 2014- & DO \\
\hline 18. Zanzamapp & Trap and report on mosquitoes in Italy & Europe & 2016- & DO \\
\hline 19. Invasive Mosquito Project & Track invasive mosquito species across the US & US & 2015- & DO \\
\hline \multicolumn{5}{|l|}{ Collaborative research $(n=32)$} \\
\hline $\begin{array}{l}\text { 1. Maori response to Christchurch } \\
\text { earthquakes }\end{array}$ & Understand how cultural attributes inform preparedness strategies & Australia & $\begin{array}{l}2010- \\
15\end{array}$ & EQ \\
\hline $\begin{array}{l}\text { 2. Environmental Competency } \\
\text { Groups }\end{array}$ & Demonstrate a method for collaborative investigation & Europe & $2007-$ & $\mathrm{FL}$ \\
\hline 3. Flood Network & Create flood detection network in the United Kingdom & Europe & 2014- & $\mathrm{FL}$ \\
\hline $\begin{array}{l}\text { 4. Participatory water monitoring in } \\
\text { Tanzania }\end{array}$ & Villagers collect and analyze data to address flood concerns & Africa & $\begin{array}{l}2001- \\
11\end{array}$ & $\mathrm{FL}$ \\
\hline $\begin{array}{l}\text { 5. Environmental exposure survey, } \\
\text { Atlanta }\end{array}$ & Document asthma and exposures in two flood-prone communities & US & 2014 & $\mathrm{FL}$ \\
\hline 6. Beacon of Hope M.O.D.E.L., & Map recovery needs using community-led recovery framework & US & 2006- & $H R$ \\
\hline
\end{tabular}


Table 2 Disaster citizen science projects grouped by citizen science model (Continued)

\begin{tabular}{|c|c|c|c|c|}
\hline Project name & Description of citizen science or volunteer activities & Location & Dates & Disaster \\
\hline 7. Community mapping post-Katrina & Pastors address uneven redevelopment patterns post-Katrina & US & 2007 & HR \\
\hline $\begin{array}{l}\text { 8. Health care needs in New Orleans } \\
\text { post-Katrina }\end{array}$ & Engage community to understand healthcare needs post-disaster & US & 2006 & HR \\
\hline $\begin{array}{l}\text { 9. Participatory action research post- } \\
\text { Katrina }\end{array}$ & Use participatory photo approach to assess health experiences & US & $\begin{array}{l}2006- \\
09\end{array}$ & $H R$ \\
\hline $\begin{array}{l}\text { 10. Videovoice for recovery post- } \\
\text { Katrina }\end{array}$ & Use participatory video approach to address issues of concern & US & $\begin{array}{l}2007- \\
08\end{array}$ & $H R$ \\
\hline $\begin{array}{l}\text { 11. Participatory research after } \\
\text { Hurricane Floyd }\end{array}$ & Develop a survey to document displaced survivor experiences & US & $\begin{array}{l}2000- \\
01\end{array}$ & $H R$ \\
\hline $\begin{array}{l}\text { 12. PhotoVoice for disaster reduction } \\
\text { strategies }\end{array}$ & Use participatory photo approach for vulnerability assessments & US & -2013 & TS \\
\hline $\begin{array}{l}\text { 13. Lake Winnipeg citizen science } \\
\text { initiative }\end{array}$ & Monitoring algal bloom formation in Lake Winnipeg, Canada & N Am & $2016-$ & $\mathrm{HAB} / \mathrm{CB}$ \\
\hline $\begin{array}{l}\text { 14. Lake Champlain volunteer } \\
\text { monitoring }\end{array}$ & Document algal blooms in Lake Champlain & US & 2004- & $\mathrm{HAB} / \mathrm{CB}$ \\
\hline 15. Appalachian Water Watch & Report emergency water pollution events & US & 2013- & $\mathrm{HAB} / \mathrm{CB}, \mathrm{CH}$ \\
\hline $\begin{array}{l}\text { 16. Participatory action research in } \\
\text { Australia }\end{array}$ & Investigate pandemic influenza risk in Indigenous communities & Australia & 2007 & DO \\
\hline 17. Mosquito Alert (Hong Kong) & Track mosquitos, breeding sites, and validate photos & Asia & $2017-$ & DO \\
\hline $\begin{array}{l}\text { 18. Understanding fishing } \\
\text { communities }\end{array}$ & Address oil spill risks in Vietnamese-American fishing communities & US & $2017-$ & $\mathrm{CH}$ \\
\hline $\begin{array}{l}\text { 19. Consortium for oil spill exposure } \\
\text { pathways }\end{array}$ & Address oil spill risks in Vietnamese-American fishing communities & US & 2015- & $\mathrm{CH}$ \\
\hline $\begin{array}{l}\text { 20. Monitoring oil contamination in } \\
\text { Louisiana }\end{array}$ & Develop a citizen science oil spill monitoring program & US & $2017-$ & $\mathrm{CH}$ \\
\hline 21. Oil Spill Crisis Map & Report and map impacts of Deepwater Horizon oil spill & US & 2010- & $\mathrm{CH}$ \\
\hline 22. Flint water crisis & Test tap water for lead contamination in Flint, Michigan & US & $\begin{array}{l}2015- \\
17\end{array}$ & $\mathrm{CH}$ \\
\hline 23. Love Canal contamination & Perform health surveys to assess chemical contamination & US & $\begin{array}{l}1978- \\
80\end{array}$ & $\mathrm{CH}$ \\
\hline 24. The Buffalo Lupus project & Assess links between waste site exposure and autoimmune disease & US & $\begin{array}{l}2001- \\
06\end{array}$ & $\mathrm{CH}$ \\
\hline $\begin{array}{l}\text { 25. Graniteville recovery \& chlorine } \\
\text { epidemiology }\end{array}$ & Address community recovery of Graniteville, SC post-chlorine spill & US & $\begin{array}{l}2005- \\
15\end{array}$ & $\mathrm{CH}$ \\
\hline $\begin{array}{l}\text { 26. Tonawanda Coke Corporation } \\
\text { pollution }\end{array}$ & Address exposure and health impacts resulting from pollution & US & $\begin{array}{l}2005- \\
09\end{array}$ & $\mathrm{CH}$ \\
\hline 27. Safecast & Map global radiation and build worldwide sensor network & Global & $2011-$ & NR \\
\hline $\begin{array}{l}\text { 28. Citizen Radioactivity Measuring } \\
\text { Stations }\end{array}$ & Take radiation measurements and make judgments on risks & Asia & $2011-$ & NR \\
\hline $\begin{array}{l}\text { 29. Towa Organic Village, Japan and } \\
\text { Fukushima }\end{array}$ & Villagers monitor radiation and perform collaborative research & Asia & $2011-$ & NR \\
\hline $\begin{array}{l}\text { 30. Nuclear Risk Management for } \\
\text { Native Communities }\end{array}$ & Address nuclear contamination impacts in tribal communities & US & $\begin{array}{l}1994- \\
04\end{array}$ & NR \\
\hline 31. St. Louis baby tooth survey & Examine radioactive material absorbed into teeth of children & US & $\begin{array}{l}1958- \\
70\end{array}$ & NR \\
\hline $\begin{array}{l}\text { 32. Hazelwood Mine fire recovery } \\
\text { effort }\end{array}$ & Develop citizen science environmental monitoring program & Australia & 2014- & $\mathrm{EF}$ \\
\hline \multicolumn{5}{|l|}{ Collegial research $(n=8)$} \\
\hline 1. Groninger Soil Movement & Monitor earthquakes and damage due to gas extraction & Europe & 2009- & EQ \\
\hline 2. Queensland Floods & Use social media to provide data and reconstruct flood extents & Australia & 2010 & $\mathrm{FL}$ \\
\hline $\begin{array}{l}\text { 3. Historic Extreme Weather Event } \\
\text { Reporting }\end{array}$ & Research historical documents on extreme weather events & N Am & 2016- & SW,HR,TD \\
\hline $\begin{array}{l}\text { 4. Community water testing in } \\
\text { Puerto Rico }\end{array}$ & Perform water testing in Puerto Rico post-Hurricane Maria & US & $2017-$ & HR \\
\hline 5. VGI and Santa Barbara wildfires & Map and share social media data during 2007-09 wildfires & US & $\begin{array}{l}2008- \\
09\end{array}$ & WF \\
\hline 6. Gulf Oil Mapping Project & Map impacts after Deepwater Horizon oil spill & US & 2010 & $\mathrm{CH}$ \\
\hline
\end{tabular}


Table 2 Disaster citizen science projects grouped by citizen science model (Continued)

\begin{tabular}{|c|c|c|c|c|}
\hline Project name & Description of citizen science or volunteer activities & Location & Dates & Disaster \\
\hline 7. iWitness Pollution Map & Report and map chemical accident reports and impacts & US & $2010-$ & $\mathrm{CH}$ \\
\hline 8. Young Crowd & Assess disaster preparedness of youth environments & Europe & $\begin{array}{l}2016- \\
17\end{array}$ & $\mathrm{AH}$ \\
\hline
\end{tabular}

Abbreviations: S Am South America, N Am North America, EQ earthquake, CL climate change, FL flooding, SW severe weather, $H R$ hurricane, $H A B / C B$ harmful algal blooms/cyanobacteria, $D R$ drought, $T D$ tornado, $L S$ landslide, DO disease outbreak, WF wildfire, VL volcanic activity, $C H$ chemical, NR nuclear radiation, $T R$ terrorism, TS tsunami, EF explosion/fire, $A H$ all hazards

References: See (Additional file 3: Table S3) for full project inventory and source references

projects were categorized into one of five citizen science models:

- Distributed or volunteer sensing $(n=19 ; 9 \%)$. Citizen scientists volunteer resources or space to facilitate data collection or analyses led by professional scientists.

- Contributory $(\boldsymbol{n}=\mathbf{9 8} ; \mathbf{4 7 \%})$. Citizen scientists collect data to assist research led by professional scientists.

- Distributed intelligence $(n=52 ; 25 \%)$. Citizen scientists perform data analyses or interpretation.

- Collaborative research $(n=32 ; 15 \%)$. Citizen and professional scientists collaborate in areas beyond data collection or analysis (e.g., problem definition, study design).

- Collegial research $(n=8 ; 4 \%)$. Citizen scientists lead research with little collaboration with professional scientists.

The next sections describe how these models of citizen science vary across project characteristics (see Table 3 for descriptive statistics).

\section{Citizen science models by disaster type}

Overall, citizen science projects were carried out most frequently for earthquakes $(n=61 ; 29 \%)$, floods $(n=52$; $25 \%)$, and hurricanes $(n=36 ; 17 \%)$. Disaster types varied across citizen science model. Earthquakes comprised the bulk of distributed sensing projects $(n=18 ; 95 \%)$. Earthquakes $(n=31 ; 32 \%)$ and floods $(n=36 ; 37 \%)$ were the main disasters for contributory projects. The majority of distributed intelligence projects focused on earthquakes ( $n=10 ; 19 \%)$, floods $(n=11 ; 21 \%)$, or hurricanes $(n=$ 16; $31 \%)$. Most collaborative research projects were focused on nuclear radiation $(n=5 ; 16 \%)$, hurricanes $(n=$ 6 ; $19 \%)$, and chemical contamination events $(n=10$; $31 \%)$. Finally, half of collegial research projects addressed either hurricane $(n=2 ; 25 \%)$ or chemical contamination events $(n=2 ; 25 \%)$.

\section{Citizen science models by lead and collaborating entities}

Most projects were led by academic/research groups ( $n=94 ; 45 \%)$ followed by government $(n=55 ; 26 \%)$, technology groups (organizations focused on development or deployment of technological resources, such as equipment or online platforms) $(n=51 ; 24 \%)$, and advocacy organizations $(n=23 ; 11 \%)$. We also collected the names of listed partners for each project, but it was often difficult to determine the role of every partner or the extent of their involvement. The majority of projects $(n=160 ; 77 \%)$ listed at least one partner. When identified, partners provided different types of services or support including: funding, technical assistance, equipment, digital platforms, manpower, administrative support, or evaluation capabilities.

Across models, academic groups led a large proportion of distributed sensing $(n=18 ; 95 \%)$, contributory $(n=$ 49 ; $50 \%)$, and collaborative research $(n=15 ; 47 \%)$ projects. Government was primarily involved as lead for contributory projects $(n=46 ; 47 \%)$. Technology groups led the greatest proportion of distributed intelligence projects $(n=34 ; 65 \%)$. Advocacy organizations showed a greater lead role in collaborative $(n=12 ; 38 \%)$ and collegial research $(n=4 ; 50 \%)$ projects compared to the other models. Finally, partnerships led 19\% $(n=6)$ of collaborative research projects, with academic and community organizations or a coalition of community groups most often comprising the partnership.

\section{Citizen science models by disaster phase}

Projects covered all disaster phases includingpreparedness $(n=135 ; 65 \%)$, response $(n=52 ; 25 \%)$, and recovery ( $n=105 ; 50 \%)$, and some covered more than one phase. Distributed sensing was more likely to be focused on preparedness $(n=17 ; 89 \%)$ and recovery $(n=15 ; 79 \%)$ versus response $(n=2 ; 11 \%)$. Contributory projects focused on preparedness $(n=81 ; 83 \%)$ and recovery $(n=$ $49 ; 50 \%)$. In contrast, the distributed intelligence model was most often used for response $(n=34 ; 65 \%)$. The recovery phase comprised $75 \%(n=24)$ of collaborative research projects compared to $50 \%(n=16)$ for preparedness and $9 \%(n=3)$ for response. Most collegial research projects focused on recovery $(n=5 ; 63 \%)$.

\section{Citizen science models by geographic setting}

Projects were implemented globally, with $40 \%(n=84)$ of projects set in the U.S. and $54 \%(n=112)$ implemented outside the U.S. Thirteen $(6 \%)$ projects were 
global in nature with no specific focus on any one country or region of the world.

Distributed sensing projects showed greater international versus U.S. prevalence $(n=9 ; 47 \%$ and $n=5$; $26 \%$, respectively), while contributory projects were more evenly distributed across U.S. and international settings ( $n=44 ; 45 \%$ and $n=49 ; 50 \%$, respectively). For distributed intelligence, projects were more prevalent internationally $(n=40 ; 77 \%)$ than in the U.S. $(n=10$; $19 \%)$. In contrast, collaborative research was more prevalent in U.S. $(n=21 ; 66 \%)$ versus international projects $(n=10 ; 31 \%)$. Collegial research projects were distributed evenly across U.S. and international settings. We also noted a few regional patterns. In the U.S., the contributory model comprised most projects across regions, with one exception. In the southeast, collaborative research was the most prevalent model type $(n=13$; $48 \%)$. Internationally, the contributory model comprised the majority of projects in Europe $(n=37 ; 69 \%)$ and Australia $(n=3 ; 30 \%)$. However, for all other continents, distributed intelligence was most prevalent.

\section{Disaster citizen science technologies}

The majority of projects $(n=159 ; 76 \%)$ required an internet-connected device to perform research. Most frequently used technologies included: crowdsourcing applications $(n=94 ; 45 \%)$; cameras or video $(n=59 ; 28 \%)$; sampling, monitoring, or sensor equipment $(n=49$; $23 \%)$; online survey tools $(n=44 ; 21 \%)$; and mapping platforms $(n=38 ; 18 \%)$.

Distributed sensing projects relied heavily on sensor equipment ( $n=17 ; 89 \%)$. The majority of contributory projects used cameras or video $(n=40 ; 41 \%)$, crowdsourcing data reporting applications $(n=39 ; 40 \%)$, or online surveys $(n=38 ; 39 \%)$. Distributed intelligence projects used crowdsourcing data analysis applications $(n=40 ; 77 \%)$ and mapping technologies $(n=32 ; 62 \%)$. For collaborative research, sampling equipment $(n=12$; $38 \%)$ and cameras or video $(n=7 ; 22 \%)$ were the most prevalent technologies. Finally, most collegial research projects used crowdsourcing reporting applications $(n=$ $5 ; 63 \%)$ and cameras or video $(n=5 ; 63 \%)$.

\section{Disaster citizen science trends}

Figure 2 displays the incidence of disaster citizen science projects across years, and shows an upward trend beginning in the late 2000s for all models. Contributory and distributed intelligence models showed similar trends around the same time period. For the 164 projects with both start and end date information, 67 (41\%) had ended while 97 (59\%) were ongoing at the time of data capture. Across models, 79\% $(n=15)$ of distributed sensing projects were ongoing, compared to $51 \%(n=50)$ for contributory, $25 \%(n=13)$ for distributed intelligence, $47 \%$ $(n=15)$ for collaborative research, and $50 \%(n=4)$ for collegial research. Projects lasted from as little as a few weeks to as long as almost 58 years. For concluded projects, average duration was 1.3 years with a range of less than a year to 12 years.

\section{Discussion}

Disaster citizen science is a rich field, comprised of diverse projects addressing many types of disasters and disaster phases. The field is growing worldwide, fueled by the use of digital technologies, and attracting multiple types of participants, including citizen volunteers, academics, government, and technology and advocacy sectors. Below we discuss four themes that arose from our assessment of the inventory. Patterns indicated potential differences across citizen science models in terms of: (1) addressing different disaster types; (2) enhancing activities across disaster phases; (3) use of technologies; and (4) exhibiting sustainability over time.

\section{Citizen science models and disaster type}

Inventory analysis revealed patterns regarding the types of disasters addressed across different citizen science models. Distributed sensing, contributory, and distributed intelligence models were mainly focused on three disaster types - earthquakes, floods, and hurricanes. In these models, which are primarily led by professional scientists, citizen scientists were involved primarily in data collection or analysis, allowing for activities such as monitoring for an event, investigating disaster impacts, or providing information to aid response.

In contrast, collaborative and collegial research models, where citizen scientists have greater roles in leading, designing, and implementing activities, showed a larger focus on chemical contamination events - a type of technological disaster. Technological disasters are anthropogenic in origin and caused by the failure of manmade systems [17-19]. Research indicates that these events are characterized by a prolonged duration, uncertain effects; distrust in authorities; and identifiable parties to blame [20]. Chemical contamination disasters may be more likely to motivate community-led actions due to a perception of failure on the part of government or other institutions to protect the public from harm. As a result, beyond collecting data to aid in PHEP actions, collaborative and collegial research models may also provide a means for communities to channel frustrations, hold institutions accountable, engage in advocacy and problem-solving, and ensure involvement in decisionmaking processes. Professional scientists who engage with citizen scientists in collaborative and collegial research models may require extra training and skills beyond what is typically provided in graduate programs. Some may be reluctant to engage in these models due to 
Table 3 Frequencies of dataset characteristics by citizen science model

\begin{tabular}{|c|c|c|c|c|c|c|c|c|c|c|c|c|}
\hline & \multirow{2}{*}{\multicolumn{2}{|c|}{$\begin{array}{l}\text { Overall } \\
209\end{array}$}} & \multicolumn{2}{|c|}{$\begin{array}{l}\text { Distributed } \\
\text { sensing }\end{array}$} & \multicolumn{2}{|c|}{ Contributory } & \multicolumn{2}{|c|}{$\begin{array}{l}\text { Distributed } \\
\text { intelligence }\end{array}$} & \multicolumn{2}{|c|}{$\begin{array}{l}\text { Collaborative } \\
\text { research }\end{array}$} & \multicolumn{2}{|c|}{$\begin{array}{l}\text { Collegial } \\
\text { research }\end{array}$} \\
\hline & & & 19 & $(9 \%)$ & 98 & $(47 \%)$ & 52 & $(25 \%)$ & 32 & $(15 \%)$ & 8 & $(4 \%)$ \\
\hline \multicolumn{13}{|l|}{ Disaster } \\
\hline Earthquake & 61 & $(29 \%)$ & 18 & $(95 \%)$ & 31 & $(23 \%)$ & 10 & $(17 \%)$ & 1 & $(3 \%)$ & 1 & $(10 \%)$ \\
\hline Flood & 52 & $(25 \%)$ & & & 36 & $(26 \%)$ & 11 & $(19 \%)$ & 4 & $(12 \%)$ & 1 & $(10 \%)$ \\
\hline Hurricane, typhoon, cyclone & 36 & $(17 \%)$ & & & 12 & $(9 \%)$ & 16 & $(28 \%)$ & 6 & $(18 \%)$ & 2 & $(20 \%)$ \\
\hline Harmful algal blooms/cyanobacteria & 18 & $(9 \%)$ & & & 10 & $(7 \%)$ & 5 & $(9 \%)$ & 3 & (9\%) & & \\
\hline Severe/extreme weather & 15 & $(7 \%)$ & & & 12 & $(9 \%)$ & 2 & $(3 \%)$ & & & 1 & $(10 \%)$ \\
\hline Climate change or sea level rise & 15 & $(7 \%)$ & 1 & $(5 \%)$ & 12 & $(9 \%)$ & 2 & $(3 \%)$ & & & & \\
\hline Chemical contamination events & 14 & $(7 \%)$ & & & 2 & $(1 \%)$ & & & 10 & $(30 \%)$ & 2 & $(20 \%)$ \\
\hline Disease outbreak & 11 & $(5 \%)$ & & & 4 & $(3 \%)$ & 5 & $(9 \%)$ & 2 & $(6 \%)$ & & \\
\hline Nuclear radiation & 8 & $(4 \%)$ & & & 2 & $(1 \%)$ & 1 & $(2 \%)$ & 5 & $(15 \%)$ & & \\
\hline Drought & 6 & $(3 \%)$ & & & 4 & $(3 \%)$ & 2 & $(3 \%)$ & & & & \\
\hline Mud/landslides & 4 & $(2 \%)$ & & & 4 & $(3 \%)$ & & & & & & \\
\hline Tornado & 4 & $(2 \%)$ & & & 3 & $(2 \%)$ & & & & & 1 & $(10 \%)$ \\
\hline Volcanic activity & 4 & $(2 \%)$ & & & 2 & $(1 \%)$ & 2 & $(3 \%)$ & & & & \\
\hline Wildfire & 3 & $(1 \%)$ & & & 2 & $(1 \%)$ & & & & & 1 & $(10 \%)$ \\
\hline Tsunami & 2 & $(1 \%)$ & & & & & 1 & $(2 \%)$ & 1 & $(3 \%)$ & & \\
\hline \multicolumn{13}{|l|}{ Lead entity } \\
\hline Academic/research & 94 & $(45 \%)$ & 18 & $(95 \%)$ & 49 & (39\%) & 10 & $(18 \%)$ & 15 & $(33 \%)$ & 2 & $(18 \%)$ \\
\hline Government & 55 & $(26 \%)$ & 1 & $(5 \%)$ & 46 & $(37 \%)$ & 6 & $(11 \%)$ & 3 & $(7 \%)$ & & \\
\hline Technology & 51 & $(24 \%)$ & & & 16 & $(13 \%)$ & 34 & $(62 \%)$ & 1 & $(2 \%)$ & & \\
\hline Advocacy & 23 & $(11 \%)$ & & & 6 & $(5 \%)$ & 1 & $(2 \%)$ & 12 & $(26 \%)$ & 4 & $(36 \%)$ \\
\hline Collaboration & 13 & $(6 \%)$ & & & 5 & $(4 \%)$ & 2 & $(4 \%)$ & 6 & $(13 \%)$ & & \\
\hline Community-based services & 6 & $(3 \%)$ & & & 1 & $(1 \%)$ & & & 4 & (9\%) & 1 & (9\%) \\
\hline Individuals/loose affiliation & 5 & $(2 \%)$ & & & 1 & $(1 \%)$ & & & 2 & $(4 \%)$ & 2 & $(18 \%)$ \\
\hline Volunteer services & 3 & $(1 \%)$ & & & 1 & $(1 \%)$ & 1 & $(2 \%)$ & & & 1 & (9\%) \\
\hline Education & 3 & $(1 \%)$ & & & 1 & $(1 \%)$ & & & 1 & $(2 \%)$ & 1 & (9\%) \\
\hline \multicolumn{13}{|l|}{ Disaster phase } \\
\hline Preparedness & 135 & $(65 \%)$ & 17 & $(50 \%)$ & 81 & $(58 \%)$ & 17 & $(27 \%)$ & 16 & $(37 \%)$ & 4 & $(33 \%)$ \\
\hline Response & 52 & $(25 \%)$ & 2 & $(6 \%)$ & 10 & $(7 \%)$ & 34 & $(54 \%)$ & 3 & $(7 \%)$ & 3 & $(25 \%)$ \\
\hline Recovery & 105 & $(50 \%)$ & 15 & $(44 \%)$ & 49 & $(35 \%)$ & 12 & $(19 \%)$ & 24 & $(56 \%)$ & 5 & $(42 \%)$ \\
\hline \multicolumn{13}{|l|}{ Location } \\
\hline Global & 13 & $(6 \%)$ & 5 & $(26 \%)$ & 5 & $(5 \%)$ & 2 & $(4 \%)$ & 1 & (3\%) & & \\
\hline United States & 84 & $(40 \%)$ & 5 & $(26 \%)$ & 44 & $(45 \%)$ & 10 & $(19 \%)$ & 21 & $(66 \%)$ & 4 & $(50 \%)$ \\
\hline Northeast & 20 & $(24 \%)$ & & & 13 & $(28 \%)$ & 2 & $(15 \%)$ & 5 & $(22 \%)$ & & \\
\hline Southeast & 27 & $(32 \%)$ & & & 9 & $(19 \%)$ & 2 & $(15 \%)$ & 13 & $(57 \%)$ & 3 & $(75 \%)$ \\
\hline Midwest & 6 & $(7 \%)$ & 2 & $(40 \%)$ & 2 & $(4 \%)$ & & & 2 & (9\%) & & \\
\hline Southwest & 8 & $(10 \%)$ & & & 5 & $(11 \%)$ & 2 & $(15 \%)$ & 1 & $(4 \%)$ & & \\
\hline West & 18 & $(21 \%)$ & 2 & $(40 \%)$ & 10 & $(21 \%)$ & 3 & $(23 \%)$ & 2 & (9\%) & 1 & $(25 \%)$ \\
\hline National & 13 & $(15 \%)$ & 1 & $(20 \%)$ & 8 & $(17 \%)$ & 4 & $(31 \%)$ & & & & \\
\hline International & 112 & $(54 \%)$ & 9 & $(47 \%)$ & 49 & $(50 \%)$ & 40 & $(77 \%)$ & 10 & (31\%) & 4 & (50\%) \\
\hline North America & 11 & $(10 \%)$ & 1 & $(11 \%)$ & 2 & $(4 \%)$ & 6 & $(15 \%)$ & 1 & (9\%) & 1 & $(25 \%)$ \\
\hline South America & 6 & $(5 \%)$ & & & 1 & $(2 \%)$ & 5 & $(13 \%)$ & & & & \\
\hline
\end{tabular}


Table 3 Frequencies of dataset characteristics by citizen science model (Continued)

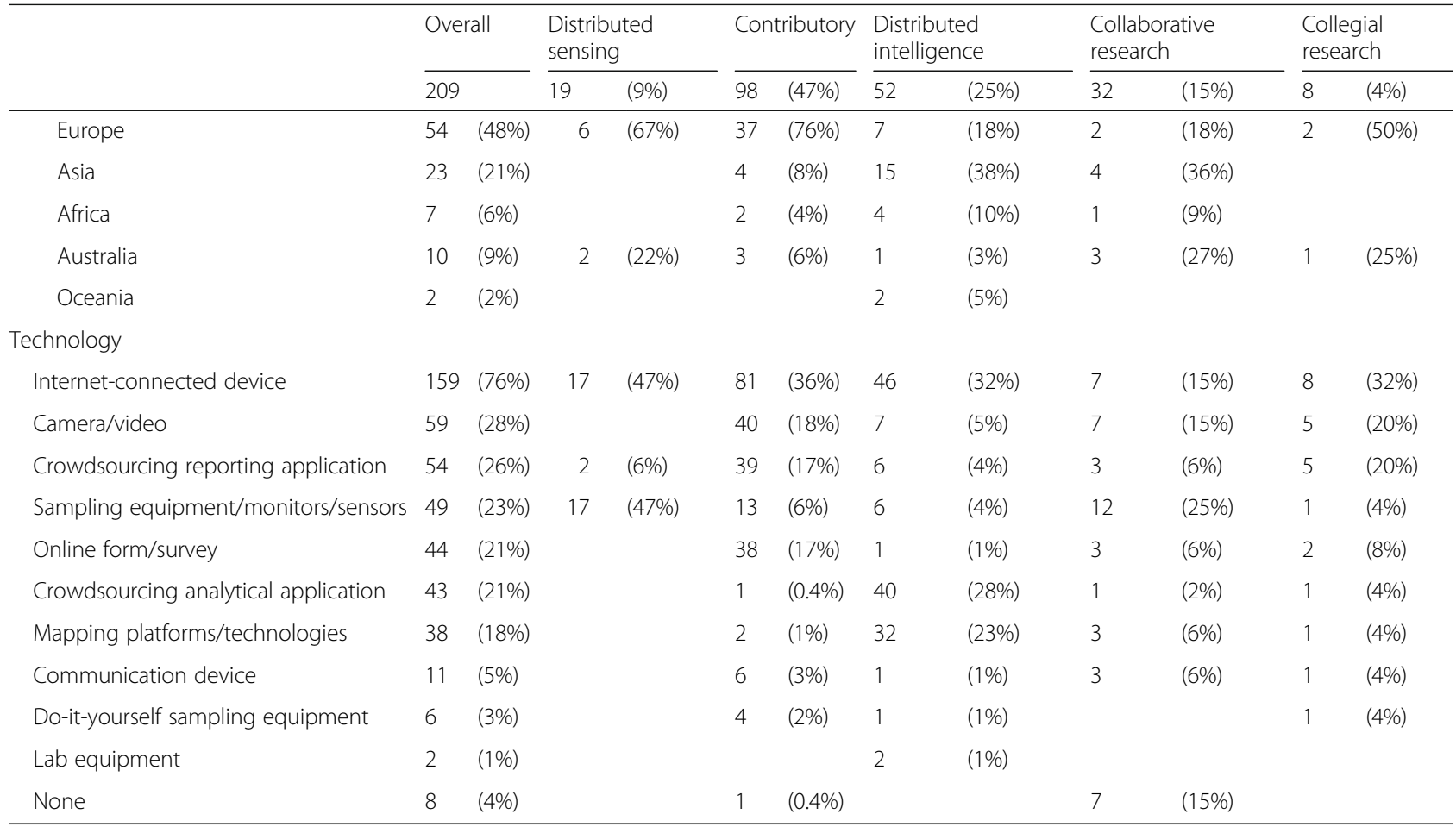

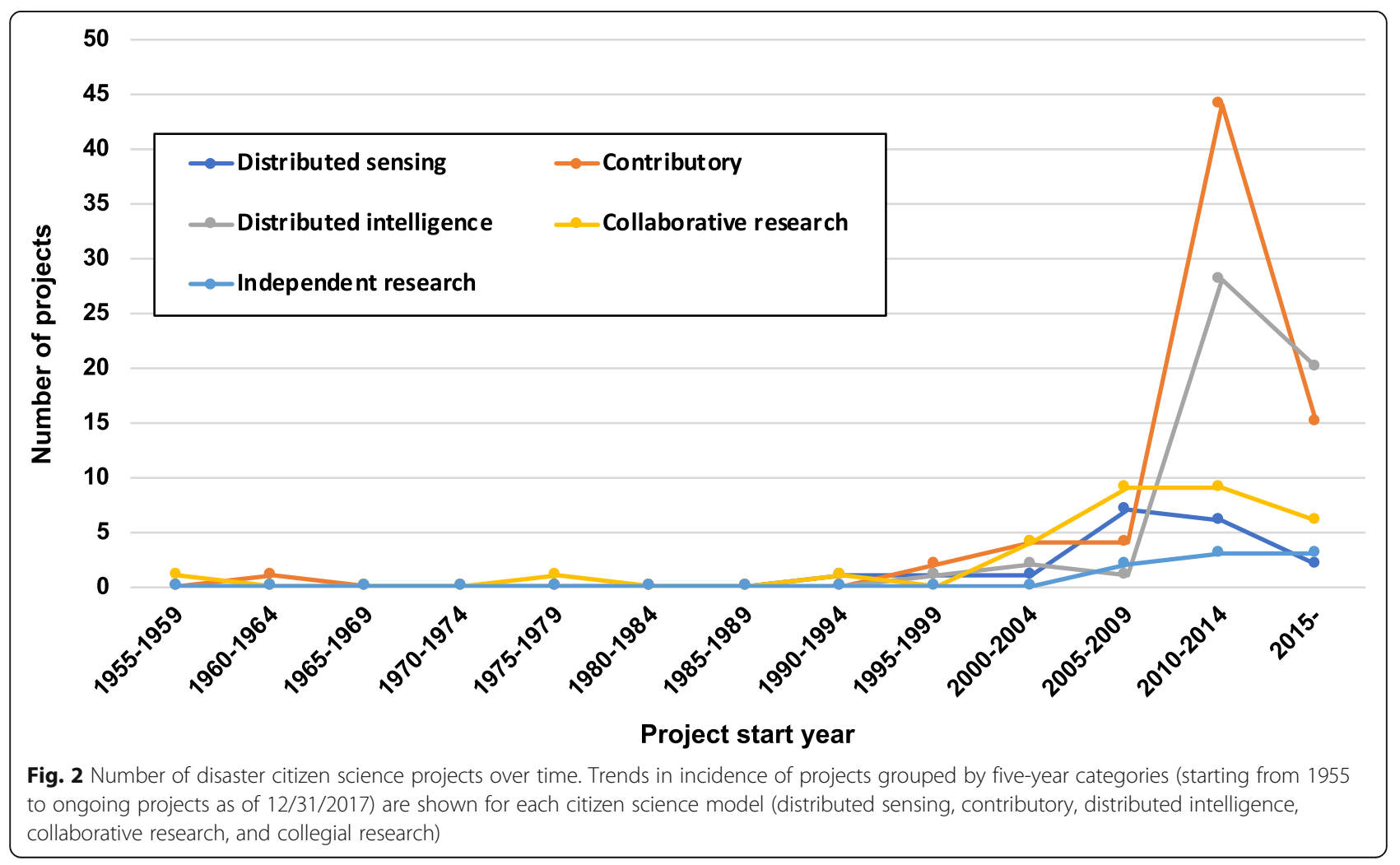


concerns that citizen scientists will have a particular agenda, limited control over data collection and quality, and the extra time and resources required to navigate complex relationships with community members and organizations [9].

\section{Citizen science models and disaster phase}

Certain models may be better suited to particular disaster phases. Distributed sensing and contributory projects focused primarily on preparedness, generally employing crowdsourced data to inform activities such as surveillance of human or environmental conditions. Given the low level of interaction and maintenance required by volunteers, distributed sensing may be a sustainable way to collect data or enhance analytical capacity.

Contributory models, along with distributed intelligence forms of citizen science, also seem wellsuited to the response phase where there is need for real-time, local information about conditions. The distributed intelligence model in particular, has allowed a new form of disaster relief operations, termed "digital humanitarianism," where volunteers away from a disaster site assist in digitally evaluating large amounts of information about the disaster (e.g., hotline requests, satellite imagery) [21]. As indicated by inventory projects, such approaches may be particularly beneficial for assisting resource-poor areas in disaster response where existing governmental or institutional structures may be inadequate to support a robust response on their own (e.g., earthquakes in Haiti, Pakistan; flooding in India, Sri Lanka; Ebola in West Africa).

Digital humanitarianism is filling a critical response need [21], but efforts are still needed to improve collection of timely, local-level data within disaster-affected areas. Federal agencies such as CDC and others have undertaken initiatives to make scientific research a part of disaster response, but there are challenges related to logistics, infrastructure, identification of research questions, and data quality [22-24]. However, inventory projects suggest that citizen science could help address some of these issues. For instance, after both the 2010 Deepwater Horizon oil spill and the 2011 Fukushima nuclear power plant meltdown, grassroots organizations led activities to create accessible tools and approaches such as apps that would allow individuals to measure and monitor disaster impacts in the environment $[25,26]$.

Finally, collaborative and collegial research projects tended to focus on the recovery phase. Collaborative research represents a somewhat more intensive project in terms of the need to develop and maintain strong partnerships, and could be highly valuable for inclusion of community needs for recovery planning and long-term recovery efforts. Given its nascency, collegial research is currently a more variable model in terms of objectives and structure. Collegial projects ranged from citizen scientists performing water quality testing to advocacy organizations spearheading crowdsourcing projects to track disaster impacts. Overall though, collegial research models afford communities the highest latitude in directing research to address community needs.

\section{Citizen science models and use of technologies}

The majority of projects used some form of digital technology, particularly smartphones with dedicated data collection applications and sharing mechanisms, and we note that the growth of disaster citizen science tracks with technological milestones such as the launch of social media ( 2004-2006) and release of mobile smartphones to the mainstream consumer market $(\sim 2007-$ 2008). According to the Pew Research Center, in 2016, $77 \%$ of Americans owned a smartphone, $73 \%$ had home broadband service, and $69 \%$ of adults reported being social media users [27]. Globally, smartphone usage in developing countries increased from $21 \%$ in 2013 to 37\% in 2015 [28]. Growing technology adoption may enhance accessibility and fuel opportunities for scientific engagement through data collection and sharing activities.

While citizen science models rely heavily on internetconnected devices, distributed sensing and distributed intelligence projects were reliant on a few specific types of technologies (crowdsourcing applications, sensors/ monitors). In contrast, contributory, collaborative, and collegial research projects incorporated a greater range of technologies (e.g., cameras, video, crowdsourcing applications, sampling equipment, online forms or survey tools).

Although a promising trend overall, there are reasons to be cautious about overreliance on digital technologies during disasters. Events such as Superstorm Sandy and Hurricane Harvey did not result in destruction of the communications or electronic infrastructure necessary to utilize internet-enabled devices. However, as seen in Puerto Rico following Hurricane Maria, this might not always be the case. Citizen science efforts should take into account how best to use different forms of technology to ensure resilient systems and which models may be best able to facilitate actions when technology is limited.

\section{Citizen science models and project sustainability}

Whether a project is sustained depends partly upon its intended goals. For example, $94 \%$ of distributed sensing projects were ongoing; this aligns with their focus on preparedness activities, which are often continuous in nature. In contrast, only $27 \%$ of distributed intelligence projects continued past a disaster event. However, the 
bulk of these projects were response-related and therefore time-limited in scope.

We note some cases however, where sustained efforts have grown organically and evolved over time. Community-led movements following the Deepwater Horizon oil spill and the Fukushima disaster led to the creation of Public Lab in the Gulf South [29], which now helps communities address their own scientific questions, and Safecast, which maintains the largest open dataset of background radiation measurements from across the globe [26]. Similarly, the 2010 Haiti earthquake catalyzed the digital humanitarianism movement, serving as the first case of a large-scale, collaborative effort between technology and humanitarian relief sectors [30]. These projects suggest a potential for sustainable citizen science models, where projects could shift objectives to address different disasters or disaster phases.

\section{Limitations}

Although we carried out a comprehensive scan of the literature, our searches only included projects that were described or conducted in English and we were limited to describing activities based on publicly available data. In addition, it was difficult at times to draw definitive boundaries around certain disaster citizen science concepts. For example, we excluded routine monitoring activities that were not directed towards a specific disaster event. Other exclusions that could be considered disaster citizen science included environmental justice projects that addressed toxic pollution concerns and climate change projects that focused on ecological rather than human impacts (e.g., invasive species, coastal erosion). Finally, we note two definitional limitations. First, our inclusion criteria for a disaster omitted rare, emerging, or slow-moving events (e.g., food security, antimicrobial resistance). Second, our designations of lead and partner entities might not always be accurate as it was often difficult to discern these characteristics from literature sources alone.

\section{Conclusions}

The public health impacts of disasters are significant: death, disease, injury, damage to homes and communities, and adverse mental and physical consequences. Given disasters will continue to strike, public health agencies are in need of tools to support PHEP efforts. Results from this first comprehensive inventory of disaster citizen science activity suggest that citizen science approaches are widely used and represent many areas of opportunity for PHEP. Disaster citizen science projects have the potential to expand PHEP capabilities such as facilitating greater data collection opportunities to support situational awareness, community risk and vulnerability assessments, and identification of recovery needs, if guidance on engaging in citizen science is made readily available to public health professionals.

The cataloguing of projects allows for a better understanding of the breadth of the field so those interested in initiating or participating in a disaster citizen science activity can find resources to tap into or leverage. Future research should explore the advantages and disadvantages of each citizen science model, barriers faced by the public health community in applying these models to different disaster contexts, promising implementation approaches, and strategies to support the proliferation of citizen science activities. In addition, more research is needed to understand the public health impacts of disaster citizen science projects, and whether and how, citizen science has demonstrably led to enhanced resilience. Our work represents a keystep in developing this understanding so that disaster citizen science achieves its potential to advance research, enhance community preparedness, and build community resilience for all.

\section{Supplementary information}

Supplementary information accompanies this paper at https://doi.org/10. 1186/s12889-019-7689-x.

Additional file 1: Table S1. Citizen science databases or websites used to identify disaster-related activities.

Additional file 2: Table S2. Search terms employed in peer-reviewed literature database searches.

Additional file 3: Table S3. Complete project inventory dataset.

\section{Abbreviations}

AH: All hazards; CDC: Centers for Disease Control and Prevention; $\mathrm{CH}$ : Chemical; CL: Climate change,; DO: Disease outbreak; DR: Drought; EF: Explosion/fire; EQ: Earthquake; FEMA: Federal Emergency Management Agency; FL: Flooding; HAB/CB: Harmful algal blooms/cyanobacteria; HR: Hurricane; LS: Landslide; N Am: North America; NR: Nuclear radiation; S Am: South America; SW: Severe weather; TD: Tornado; TR: Terrorism; TS: Tsunami; VL: Volcanic activity; WF: Wildfire

\section{Acknowledgements}

The authors are grateful for manuscript editing contributions from Dr. Shoukat Qari at the US Centers for Disease Control and Prevention. This work was supported by the US Centers for Disease Control and Prevention through research contract 200-2016-92420.

\section{Authors' contributions}

$\mathrm{RC}$ led the design and conceptualization of the project, carried out project screenings and data extractions, and led data interpretation and manuscript drafting activities. EPS and SA were involved in analytical design, carried out project screenings, extracted data for the inventory, and were major contributors in data interpretation and manuscript drafting. ML was involved in the design and conceptualization of the project and contributed substantially to data interpretation and manuscript development and revision. VK and JM helped to identify and screen projects and contributed to manuscript development and revision. ET and EC contributed substantially to data interpretation and manuscript development and revision. LUP was substantially involved in the design and conceptualization of the project, extracted data for the inventory, and contributed to manuscript development and revision. All authors have read and approved the final version of the manuscript. 


\section{Funding}

This work was funded by the US Centers for Disease Control and Prevention (CDC) through research contract 200-2016-92420. The manuscript was authored by both RAND and CDC authors; thus, the funder was involved in all aspects of the work, including study design, data collection, analysis and interpretation, and manscuript writing and revision.

\section{Availability of data and materials}

All data generated or analyzed during this study are included in this published article [and its additional information files].

\section{Ethics approval and consent to participate}

Not applicable.

\section{Consent for publication}

Not applicable.

\section{Competing interests}

The authors declare that they have no competing interests. The findings and conclusions in this report are those of the authors and do not necessarily represent the official position of the Centers for Disease Control and Prevention.

\section{Author details}

${ }^{1}$ RAND Corporation, 1200 South Hayes Street, Arlington, VA 22202, USA. ${ }^{2}$ RAND Corporation, 1776 Main Street, Santa Monica, CA 90401, USA. ${ }^{3}$ Center for Preparedness and Response, Centers for Disease Control and Prevention, 2877 Brandywine Road, MS K-72, Atlanta, GA 30341, USA. ${ }^{4}$ Center for Preparedness and Response, Centers for Disease Control and Prevention, 1600 Clifton Road NE, MS K-72, Atlanta, GA 30333, USA.

Received: 13 February 2019 Accepted: 25 September 2019

Published online: 23 October 2019

\section{References}

1. Centers for Disease Control and Prevention. Public health preparedness capabilities: national standards for state and local planning. 2011. https:// www.cdc.gov/phpr/readiness/capabilities.htm. Accessed 15 Oct 2018.

2. Federal Emergency Management Agency. A whole community approach to emergency management: principles, themes, and pathways for action. 2011. https://www.fema.gov/media-library-data/20130726-1813-25045-3330/ whole_community_dec2011____pdf.

3. Office of the Assistant Secretary for Preparedness and Response. National Health Security Strategy and Implementation Plan 2015-2018. 2015. https:// www.phe.gov/Preparedness/planning/authority/nhss/Pages/strategy.aspx. Accessed 15 Oct 2018

4. Centers for Disease Control and Prevention. Preparedness, PREP-15. In: Healthy People 2020. 2018. https://www.healthypeople.gov/2020/topicsobjectives/topic/preparedness/objectives.

5. Office of the Assistant Secretary for Preparedness and Response. Bystanders as first responders. https://www.phe.gov/eccc/PCCC/Pages/bystanders.aspx. Accessed 24 Aug 2018.

6. Gursky EA, Bice G. Assessing a decade of public health preparedness: progress on the precipice? Biosecur Bioterror. 2012;10:55-65.

7. Eitzel MV, Cappadonna JL, Santos-Lang C, Duerr RE, Virapongse A, West SE, et al. Citizen science terminology matters: exploring key terms. Citizen Science: Theory and Practice. 2017;2:1-20.

8. Chari R, Matthews $\sqcup$, Blumenthal MS, Edelman AF, Jones T. The promise of community citizen science. RAND Corporation. 2017. https://www.rand.org/ pubs/perspectives/PE256.html.

9. Miller-Rushing A, Primack R, Bonney R. The history of public participation in ecological research. Frontiers Ecol Environ. 2012;10:285-90.

10. Office of the Assistant Secretary for Preparedness and Response. Public health emergency declarations. 2018. https://www.phe.gov/emergency/ news/healthactions/phe/Pages/default.aspx. Accessed 24 Aug 2018.

11. Federal Emergency Management Agency. Disasters. 2018. https://www. fema.gov/disasters.

12. Busby JW. Climate change and national security: an agenda for action. Council on Foreign Relations 2007. https://www.cfr.org/report/climatechange-and-national-security. .
13. Wilderman CC. Models of community science: design lessons from the field. Presented at the Citizen Science Toolkit Conference, Ithaca, N.Y., June 2023. 2007. http://www.informalscience.org/models-community-sciencedesign-lessons-field.

14. Shirk JL, Ballard HL, Wilderman CC, Phillips T, Wiggins A, Jordan R, et al. Public participation in scientific research: a framework for deliberate design. Ecol Soc. 2012;17:29.

15. Chandra A, Acosta JD, Howard S, Uscher-Pines L, Williams MV, Yeung D, et al. Building community resilience to disasters: a way forward to enhance national health security. RAND Corporation 2011. https://www.rand.org/ pubs/technical_reports/TR915.html.

16. Haklay M. Citizen science and volunteered geographic information overview and typology of participation. In: Sui DZ, Elwood S, Goodchild MF, editors. Crowdsourcing geographic knowledge: volunteered geographic information (VGI) in theory and practice. Berlin: Springer; 2013. p. 105-22.

17. Erikson K. A new species of trouble: the human experience of modern disasters. New York: W.W. Norton and Company; 1995.

18. Gill DA, Picou JS. Technological disaster and chronic community stress. Soc Nat Resour. 2008;11:795-815.

19 Adeola FO. Industrial disasters, toxic waste, and community impact: health effects and environmental justice struggles around the globe. Lanham: Lexington Books; 2012.

20. Palinkas LA. A conceptual framework for understanding the mental health impacts of oil spills: lessons from the Exxon Valdez oil spill. Psychiatry. 2012; 75:203-22.

21. Digital Humanitarian Network. About. http://digitalhumanitarians.com 2018. Accessed 24 Aug 2018.

22. National Institute for Occupational Safety and Health. Disaster science responder research program. 2018. https://www.cdc.gov/niosh/topics/ disasterscience. Accessed 24 Aug 2018.

23. National Institutes of Health. NIH disaster research response (DR2). 2018. https://dr2.nlm.nih.gov. Accessed 24 Aug 2018

24. Office of the Assistant Secretary for Preparedness and Response. Science preparedness. 2015. https://www.phe.gov/Preparedness/planning/science/ Pages/default.aspx. Accessed 24 Aug 2018.

25. Grassroots mapping: Gulf oil spill mapping. http://grassrootsmapping.org/ gulf-oil-spill 2010. Accessed 24 Aug 2018.

26. Safecast. Open environmental data for everyone. 2018. https://blog.safecast. org. Accessed 24 Aug 2018.

27. Pew Research Center. Record shares of Americans now own smartphones, have home broadband. 2017. http://www.pewresearch.org/fact-tank/2017/ 01/12/evolution-of-technology. Accessed 24 Aug 2018.

28. Pew Research Center. 10 facts about smartphones as the iPhone turns 10. 2017. http://www.pewresearch.org/fact-tank/2017/06/28/10-facts-aboutsmartphones. Accessed 24 Aug 2018.

29. Public Lab. https://publiclab.org 2018.

30. Meier P, Munro R. The unprecedented role of SMS in disaster response: learning from Haiti. SAIS Rev. 2010:30:91-103.

\section{Publisher's Note}

Springer Nature remains neutral with regard to jurisdictional claims in published maps and institutional affiliations.

Ready to submit your research? Choose BMC and benefit from:

- fast, convenient online submission

- thorough peer review by experienced researchers in your field

- rapid publication on acceptance

- support for research data, including large and complex data types

- gold Open Access which fosters wider collaboration and increased citations

- maximum visibility for your research: over $100 \mathrm{M}$ website views per year

At BMC, research is always in progress.

Learn more biomedcentral.com/submission 